\title{
Acute brucellosis associated with isolated splenic and left gastric artery vasculitis and acute ischemic bowel infarction. A systematic review of the most recent cases
}

\author{
Mariam Ahmed Saad', Eiman Saeed Ahmed², Fahad Ali Alghamdi ${ }^{3}$, Yasser Ragab Fahmy ${ }^{4,5}$, \\ Yasser Emadeldeen Amin 6 , Ahmed Ahmed Saad ${ }^{2,7}$ \\ 'Department of Medical Oncology, National Cancer Institute, Cairo, Egypt; \\ ${ }^{2}$ Department of Internal Medicine Department, Dr. Erfan and Bagedo General Hospital, Jeddah, Saudi Arabia; \\ ${ }^{3}$ Department of Pathology, College of Medicine, King Abdulaziz University, Jeddah, KSA; \\ ${ }^{4}$ Department of Radiology, Faculty of Medicine Cairo University, Cairo, Egypt; \\ ${ }^{5}$ Department of Radiology, Dr. Erfan and Bagedo General Hospital, Jeddah, Saudi Arabia; \\ ${ }^{6}$ Department of Rheumatology, Faculty of Medicine Cairo University, Cairo, Egypt; \\ 'Department of Internal Medicine, Faculty of Medicine, Cairo University, Cairo, Egypt
}

Article received 30 April, 2021; accepted 28 June, 2021

\section{SUMMARY}

Brucellosis is a multisystem bacterial zoonosis caused by Gram-negative bacteria Brucella spp. Ingestion of infected food products, direct contact with an infected animal, or inhalation of aerosols are all ways for germs to spread from animals to humans. Intestinal vasculitis with gangrene due to brucellosis has rarely been reported. We report a 62-year-old male patient presenting with acute onset of recurrent attacks of abdominal pain, remittent fever, malaise, and weight loss, which were followed by severe left hypochondrium abdominal pain with rigidity and signs of acute abdomen. Brucellosis was clinically suspected and confirmed by an enzyme-linked immunosorbent assay against the Bru- cella melitensis species. An abdominal CT scan revealed isolated splenic and left gastric artery vasculitis, leading to acute bowel ischemia, bowel infarction and gangrenous jejunal bowel segment. Histopathological examination of the resected gangrenous bowel segment revealed leucocytoclastic vasculitis. The patient was successfully treated with a standardized antimicrobial therapy for brucellosis and a short course of steroids with a complete resolution of the symptoms and signs. The case is discussed and the literature is reviewed.

Keywords: Brucella melitensis, leucocytoclastic vasculitis, intestinal brucellosis, brucella intestinal vasculitis.

\section{INTRODUCTION}

$\mathrm{B}_{\mathrm{i} \mathrm{r}}^{\mathrm{r}}$ rucellosis is a multisystem common zoonotic infectious disease caused by Gram-negative intracellular bacteria found worldwide that has a broad range of characteristics, which range

Corresponding author

Yasser Emad

E-mail: yasseremad68@gmail.com from acute fever and hepatomegaly to chronic infections that most commonly affect the central nervous system, cardiovascular system, or skeletal system [1]. Later complications may include arthritis or epididymoorchitis, spondylitis, neurobrucellosis, liver abscess formation, and endocarditis. The latter is a potentially fatal disease complication [2].

After exposure to Brucella, humans generally have a two to four week latency period before 
exhibiting symptoms, which include acute undulating fever ( $>90 \%$ of all cases), headache, arthralgia $(>50 \%)$, night sweats, fatigue, and anorexia [3]. Few case reports addressed potential vascular involvement during the disease course of brucellosis [1, 4-13]. Aortitis is the most commonly reported vascular affection in brucellosis [4-6]. In brucellosis, different types of vasculitis have been reported infrequently, such as leukocytoclastic vasculitis (LCV), which refers to small blood vessel inflammation, recurrent panniculitis, mixed cryoglobulinemia with renal failure, cutaneous vasculitis and peritonitis, celiac artery (CA) and superior mesenteric artery (SMA) vasculitis, and thrombosis of the inferior vena cava (IVC) [1, 7-12].

In this report, we present an elderly male patient with brucellosis who developed acute bowel ischemia, bowel infarction, and peritonitis as a result of underlying isolated splenic and left gastric artery vasculitis, which caused acute bowel ischemia and eventually jejunal bowel segment gangrene. The resected gangrenous bowel segment was histopathologically and pathologically examined, and evidence of LCV was found. The aim of the current report is to increase awareness of this rare disease association, and to discuss the management strategy and outcome.

\section{CASE REPORT}

A 62-year-old man with a medical history of coronary artery disease (CAD), hypertension, and dyslipidemia was reported to our facility with four weeks of remittent fever and abdominal pain. He also had bouts of diarrhea, generalized arthralgia, and a general feeling of malaise. The patient also admitted to eating unpasteurized fresh cheese. A physical examination revealed high fever of $39.3^{\circ} \mathrm{C}$ and a tender abdomen on palpation, with no other significant clinical signs of medical importance. Initial laboratory investigations upon admission showed a white blood cell (WBCs) count of 4.6 (4.8-10), hemoglobin 14.5 (14.8-17) g/dl, platelets 186 (150-450), and C-reactive protein (CRP) $9.1 \mathrm{mg} / \mathrm{dl}(0-0.5)$ that increased to $21 \mathrm{mg} / \mathrm{dl}$ with a persistent fever and abdominal pain. Serum creatinine 0.9 (0.7-1.2) $\mathrm{mg} / \mathrm{dl}$, serum potassium $4.3 \mathrm{mmol} / \mathrm{L}$ (3.5-5.1), serum sodium $134 \mathrm{mmol} / \mathrm{L}$ (135-145), SGOT 21 I.U/L (5-37), SGPT 30 I.U/L (0-41), alkaline phos-

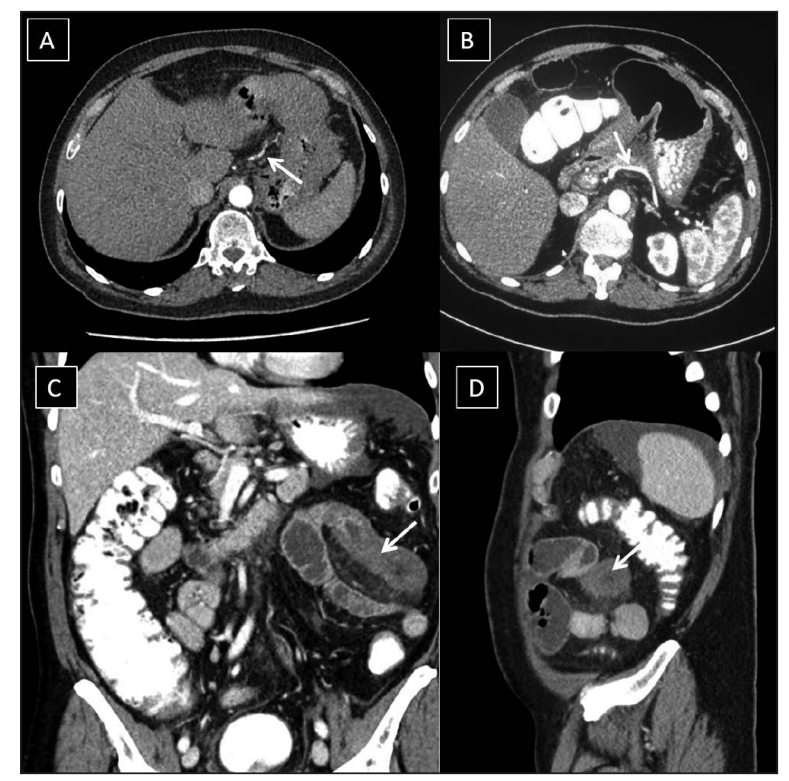

Figure 1 - (A): Abdominal computerized tomography (CT) scan with enhancement arterial phase in axial section showing left gastric artery mural irregularities and subtle mural attenuation (white arrow (B): Abdominal CT scan with enhancement arterial phase in axial section showing mural irregularities of the splenic artery (white arrow): Abdominal CT scan with enhancement arterial phase in coronal reconstruction showing left hypochondrial segmental small bowel mural thickening (white arrow) lacking contrast uptake with mesenteric fat smudging and minimal fluid collection indicative of gangrenous small jejunal bowel segment (Poor enhancement of the ischemic loop was also validated in the venous phase of CT scanning (D): Abdominal CT scan with enhancement arterial phase in sagittal reconstruction showing mural thickening of jejunal loop segment (white arrow) and non-visualized mesenteric vasculature.

phatase 89 I.U/L, LDH 170 I.U/L (100-250), and stool analysis and culture were normal. Serum amylase $68 \mathrm{~g} / \mathrm{dl}$ (28-100), lipase $27 \mathrm{~g} / \mathrm{dl}$ (5-60), albumin $4 \mathrm{~g} / \mathrm{dl}$ (3.4-4.8), direct bilirubin $0.37 \mathrm{mg} /$ $\mathrm{dl}(0-0.3)$, indirect bilirubin $0.65 \mathrm{mg} / \mathrm{dl}(0.2-1.2)$, INR is 1, PTT is 31 seconds (26-40). For many reasons, brucellosis was suspected clinically: first, because the disease is endemic in Saudi Arabia, and second, because the patient's family lives in a farm and raised goats. Therefore, brucellosis was a possible diagnosis. An enzyme-linked immunosorbent assay (ELISA) was done, which initially gave positive results with a titer of $1 / 320$ against the Brucella melitensis species, which climbed to $1 / 1280$ one week later. The patient experienced a continuous fever, as well as excruciating hypo- 
chondrial pain with rigidity and elevated markers of inflammation. An urgent abdominal computerized tomography (CT) scan with enhancement revealed mural irregularities of the splenic and left gastric arteries with subtle mural attenuation, left hypochondrial segmental small bowel mural thickening with mesenteric fat smudging and minimal fluid collection indicative of gangrenous small jejunal bowel segment (Figure 1 a-d) with no radiological evidence of abdominal or thoracic aortic artery involvement suggestive of aortitis. Subsequently, our patient was assessed by the general surgery team and underwent exploratory laparotomy with resection of the gangrenous jejunal bowel segment (Figure 2a). Histological examination of the resected gangrenous bowel segment showed foci of large mucosal ulceration associated with granulation tissue and intra-parenchymal hemorrhagic changes (Figure $2 b$ ). Many blood vessels showed a large neutrophilic infiltrate in the vessel wall, which was accompanied by karyorrhexis debris and fibrinoid necrosis (Figure 2c). The latter features were consistent with leucocytoclastic vasculitis. The patient was started on a combination therapy of streptomycin $1 \mathrm{~g}$ i.m. daily for two weeks, rifampicine 600 $\mathrm{mg}$ /day p.o. and doxycycline $100 \mathrm{mg}$ bid p.o. for 12 weeks in addition to oral prednisolone $40 \mathrm{mg}$
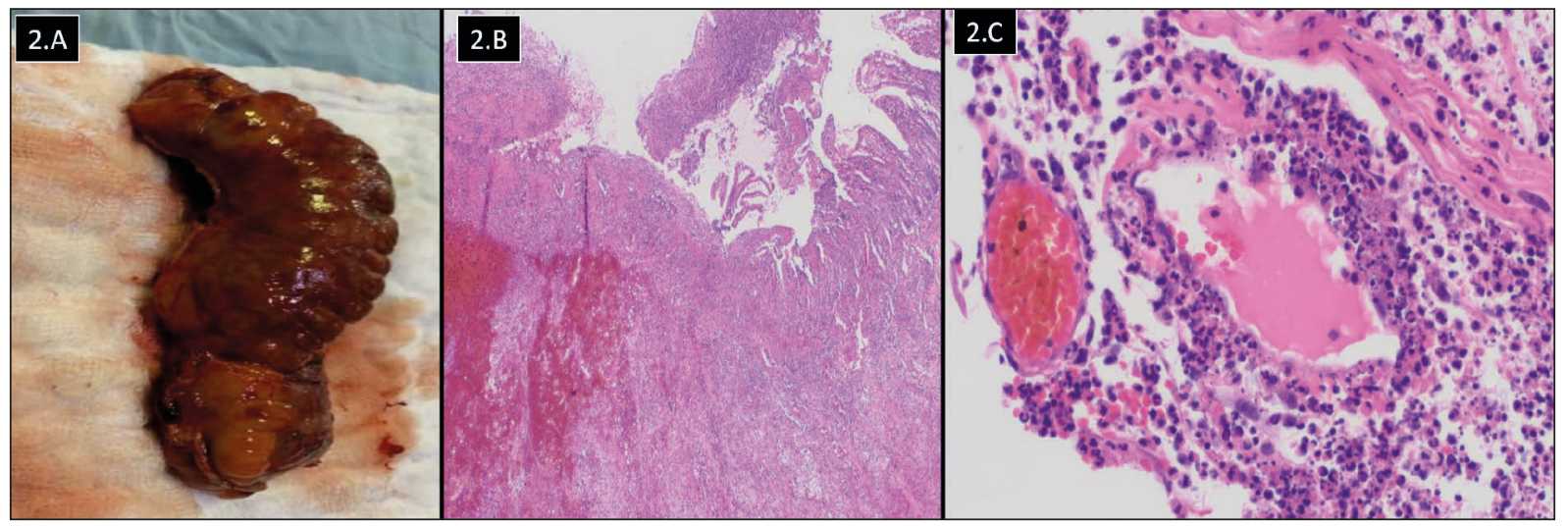

Figure 2 - (2.a) The resected gangrenous jejunal bowel segment; (2b) Small Intestine (Low power, 40x): Mucosal ulceration (top central and left side) associated with granulation tissue and intra-parenchymal hemorrhagic changes; (2c) Small blood vessels in the vicinity of the ulceration (High power; $400 \mathrm{x}$ ) with heavy neutrophilic infiltration of the wall of the vessel associated with karyorrhexis debris and fibrinoid necrosis.

Table 1 - Summary of the most recent case reports that described association between brucellosis and vascular affection.

\begin{tabular}{|l|c|c|c|c|}
\hline \multicolumn{1}{|c|}{ References } & Age & Sex & Brucella species & Vascular affection \\
\hline Current case & 61 & Male & B. melitensis & Splenic and left gastric arteries \\
\hline [1] Wang et al. 2017 & 15 & Male & Not identified & Celiac \& superior mesenteric arteries \\
\hline [5] Benedetto et al. 2011 & 81 & Male & B. melitensis & Abdominal aorta and iliac arteries \\
\hline [6] Chen et al. 2011 & 71 & Male & B. melitensis & Thoracic and abdominal aortic \\
\hline [7] Korkmaz et al. 2016 & 36 & Female & B. melitensis & Leukocytoclastic vasculitis \\
\hline [8] Uçmak et al. 2014 & 17 & Male & Not identified & Leukocytoclastic vasculitis, splenic infarction \\
\hline [9] Dizbay et al. 2007 & 64 & Male & B. melitensis & Leukocytoclastic vasculitis, renal failure, peritonitis \\
\hline [10] Tanyel et al. 2008 & 83 & Male & B. melitensis & Panniculitis \\
\hline [11] Lazcano et al. 2005 & 59 & Male & B. melitensis & $\begin{array}{c}\text { Mixed cryoglobulinemia with renal } \\
\text { failure, cutaneous vasculitis }\end{array}$ \\
\hline [12] Rüegger et al. 2017 & 23 & Female & B. melitensis & Inferior vena cava thrombosis \\
\hline
\end{tabular}


once daily for 2 weeks which was gradually tapered and stopped over four weeks. There was significant clinical improvement at regular outpatient clinic follow-up visits, and the brucella antibody titer and CRP were back to normal values. The gastrointestinal manifestations recovered and a complete cure was achieved after 12 weeks of antimicrobial treatment.

\section{Systematic literature review of most recent cases since 2005 to date}

A systematic literature review was conducted to find relevant case reports that had previously been published in this domain. To uncover similar associations, PubMed was searched with the following keywords: brucellosis/vasculitis and brucellosis/splanchnic vasculitis. Relevant previously published case reports with vascular complications in association with brucellosis are included and summarized in Table 1.

\section{DISCUSSION}

In various countries, such as the Mediterranean basin and the Arabian Peninsula, brucellosis is an endemic illness. The infection rate in Saudi Arabia is around 70 per 100,000 people [13]. Despite the fact that brucellosis affects multiple organ systems, gastrointestinal and splanchnic artery involvement with vasculitis is a rare but serious disease presentation which can result in significant morbidity and mortality [1]. In the current report, we described the clinical disease course of an elderly male patient with an established diagnosis of brucellosis complicated by splenic and left gastric arteriolitis; the latter was presented by clinical features of acute abdomen due to acute bowel ischemia that led to a gangrenous jejunal bowel segment. Likewise, Wang et al., reported a similar case of brucellosis in a 15-year-old adolescent who presented with intermittent abdominal pain, diarrhea, and fever, with intestinal tract involvement [1]. The $\mathrm{CT}$ abdomen revealed stenosis of the celiac artery (CA) and the superior mesenteric artery (SMA). Other detailed CT findings revealed a thickened bowel wall in the ileocaecal area, and terminal ileum, with an abnormal enhancement of the mucosal surface of the affected bowel segments. Furthermore, CT showed slight narrowing in the beginning of the CA, SMA stenosis, enlarged spleen with multiple wedged ar- eas of low attenuation in the spleen and the inferior pole of the left kidney that were suspected to be areas of ischemic infarction [1], Given that the splanchnic arteries may get involved in a vasculitic process during the course of brucellosis illness. According to previous research, the aorta appears to be more commonly involved in brucellosis patients [4-6]. Of note, contiguous propagation from vertebral involvement (brucellar spondylitis) was suggested as the etiological cause of brucellar aortitis [5]. Importantly brucellar aortitis can be aggressive to the extent that it can lead to aortic rupture $[5,6]$. Cascio et al. analyzed the epidemiological and clinical characteristics of 46 cases of brucellar aortic involvement [14]. The authors reported that in 18 of these cases, the complications involved the ascending thoracic aorta; 16 of these cases were related to brucellar endocarditis. In the remaining 30 cases, the complication involved the descending thoracic aorta or the abdominal aorta; in 13 of these cases, the complication was related to spondylitis of the lumbar spine [14]. The authors recommended that patients older than 50 years, who have blood cultures positive for $\mathrm{Bru}$ cella spp presenting with fever and back, abdominal, or chest pain, should undergo an extensive diagnostic test for brucellar aortic involvement and aneurysm formation. Similarly, patients diagnosed with aortic mycotic aneurysms or with pseudo-aneurysms should be screened for possible brucellosis. In patients with brucellar thoracic aortic involvement, concomitant endocarditis should be ruled out, and in the case of brucellar abdominal aortic involvement, concomitant brucellar spondylitis should be excluded. Taken together, a contrasting strategy should be applied to patients with endocarditis and/or spondylitis. Importantly, in all clinical scenarios, anti-bacterial antibiotic therapy should be initiated as soon as possible, followed by appropriate surgical or endovascular management in the case of aortic rupture $[5,6,14]$. In our patient, we excluded brucellar aortic involvement by careful interpretation of the enhanced abdominal CT study.

Furthermore, different patterns of vasculitis associated with brucellosis have been identified in the literature on a rare basis. Cutaneous Leukocytoclastic Vasculitis (LCV), which is the term used to describe small vessel vasculitis, was reported in three patients with brucellosis [7-9]. The cutaneous LCV was associated with splenic infarction 
in one patient, and enhanced abdominal CT revealed an enlarged spleen and triangular splenic infarct, and in another patient, the LCV was associated with brucellar peritonitis and renal failure, which eventually led to death $[8,9]$. Nevertheless, cryoglobulinemia has been described in many infectious diseases, i.e., brucellosis, leptospirosis, psittacosis, and Mediterranean tick typhus. In another report, a 59-year-old male was presented with mixed cryoglobulinemia with renal failure, cutaneous vasculitis and peritonitis due to Brucella melitensis, with the latter disease manifestations resolved with specific antimicrobial therapy [11]. Taken together, cutaneous LCV during the disease course of brucellosis may precede aggressive and extensive systemic vascular involvement. Of note, other cutaneous manifestations of brucellosis occur in about $6 \%-13 \%$ of patients. Other skin lesions reported in addition to cutaneous vasculitis include rashes, papules, ulcers, abscess, erythema nodosum, ecchymosed skin rash, purpura, and panniculitis [10].

In another important report, Rüegger et al. described a 23-year-old woman with a newly diagnosed thrombosis of the inferior vena cava associated with a Brucella melitensis infection [12]. The authors also reviewed another 14 previously reported patients with various major venous thrombotic events, i.e. deep venous thrombosis (DVT), internal jugular, cerebral venous sinus thrombosis (CVST), and retinal vein thrombosis. The authors suggested a possible relationship between brucellosis and the development of venous thrombotic events.

The current concept supports the fact that cardiovascular involvement is the main cause of mortality due to Brucella spp infection [12]. Cardiovascular involvement most commonly manifests as endocarditis, peripheral and cerebrovascular aneurysms, or arterial and venous thrombosis. Of note, brucellar bacteremia can lead to infective endocarditis and aortic endarteritis [15]. Brucellar endocarditis most commonly affects the aortic valve, followed by the mitral valve. It's a slow-moving, destructive condition that's often accompanied by massive vegetation. It's also linked to a high rate of intracardiac abscess and congestive heart failure [16].

The current case study shows a possible link between brucellosis and various vascular involvement patterns, with brucellar aortitis being the most significant and potentially fatal complication [4-6, 15]. Arterial as well as major venous thrombotic events can be observed during the brucellosis disease course. To the best of the authors' knowledge, splanchnic arteries may be the site of a vasculitic process that can lead to serious acute bowel ischemia and serious consequences. The latter findings are important because gastrointestinal manifestations are common as an early disease presentation in brucellosis. These symptoms can range from mild anorexia, diarrhea, or constipation to more serious complications like mesenteric lymphadenitis, liver or spleen involvement, or cholecystitis. In rare cases, life-threatening complications such as colitis, pancreatitis, peritonitis, or intestinal obstruction can occur [16]. Likewise, in a previous report and in our report, arteriolitis of the branches of the splanchnic circulation may lead to acute ischemic bowel infarction and gangrene [1]. The advantage of our report is that we established histopathological proof of underlying vasculitis of the resected gangrenous bowel segment, leaving no doubt about the aetiopathogenesis of this rare association with brucellosis. Based on the histopathological proof of vasculitis, our patient received in addition to antimicrobial therapy for 12 weeks, a course of oral prednisolone $40 \mathrm{mg}$ daily for two weeks, which was gradually tapered and stopped after four weeks. Using steroid in our patient is important if we take into consideration that LCV is mediated by immune complex deposition. In LCV, circulating antigens in the body induce antibody formation. These antibodies bind to the circulating antigens and create immune complexes, which then deposit within vessels, activating complement and inducing inflammatory mediators [17]. Steroids would control this complex autoimmune process, while antimicrobial therapy helps to eradicate the brucellar bacteremia to prevent more antigenic stimulation and eventually prevent any further burden of immune complex formation and deposition.

In conclusion, gastrointestinal manifestations are diverse and may be the only symptoms of brucellosis that occur. To rule out acute bowel ischemia due to an underlying vasculitis process, a contrast enhanced abdominal CT is required in known individuals with brucellosis manifested by persistent severe abdominal pain and rising inflammatory markers in combination with symptoms 
and signs of acute abdomen. In brucellosis, the splanchnic circulation that comprises three main divisions of the abdominal aorta, namely the celiac, superior mesenteric and inferior mesenteric arteries, could be involved in the autoimmune vasculitic process. The underlying cause of acute intestinal ischemia in our patient was documented histopathologically as LCV affecting the splenic and left gastric arteries.

\section{Conflicts of interest \\ None.}

\section{Funding}

None.

\section{REFERENCES}

[1] Wang M, Zhu Q, Yang Q, et al Intestinal brucellosis associated with celiac artery and superior mesenteric artery stenosis and with ileum mucosa and submucosa thickening: A case report. Medicine (Baltimore). 2017; 96 (2), 5893.

[2] Gorvel JP Brucella: a Mr "Hide" converted into Dr Jekyll. Microbes Infect. 2008; 10 (9), 1010-3.

[3] Atluri VL, Xavier MN, de Jong MF, den Hartigh AB, Tsolis RM. Interactions of the human pathogenic Brucella species with their hosts. Annu Rev Microbiol. 2011; 65 (1), 523-41.

[4] Tosatto V, Boattini M, Nascimento P, Moura RB. Lymphadenitis and aortitis due to Brucella melitensis infection. Infection. 2020; 48 (2), 313-4.

[5] Benedetto F, Lentini G, Passari F, et al. Endovascular repair of aortic rupture due to Brucella aortitis. Vasa. 2011; 40 (2), 150-6.

[6] Chen SL, Kuo IJ, Fujitani RM, Kabutey NK Endovascular Management of Concomitant Thoracic and
Abdominal Aortic Ruptures Resulting from Brucellosis Aortitis. Ann Vasc Surg. 2017; 38, 190.e1-190.e4 .

[7] Korkmaz P, Kıdır M, Namdar ND, Özmen A, Uyar C, Değer AN Case of brucellosis with recurrent attacks of vasculitis. Case Rep Infect Dis. 2016; 2016, 5740589.

[8] Uçmak F, Uçmak D, Beştaş R, Anli RA, Adanir H. Acute brucellosis associated with leukocytoclastic vasculitis and splenic infarct. Infez Med. 2014; 22 (4), 326-30. [9] Dizbay M, Hizel K, Kilic S, Mutluay R, Ozkan Y, Karakan T. Brucella peritonitis and leucocytoclastic vasculitis due to Brucella melitensis. Braz J Infect Dis. 2007; 11 (4), 443-4.

[10] Tanyel E, Fisgin NT, Yildiz L, Leblebicioglu H, Doganci L, Tulek N. Panniculitis as the initial manifestation of brucellosis: a case report Am J Dermatopathol . 2008; 30 (2), 169-71.

[11] Lazcano IH, Mendez LS, Santos JS. Mixed cryoglobulinemia with renal failure, cutaneous vasculitis and peritonitis due to Brucella melitensis. J Infect. 2005; 51 (5), 257-9. [12] Rüegger K, Tarr P, Karatolios K, Humburg J, Hügli $R$, Jeanneret $C$. Brucellosis and thrombosis of the inferior vena cava. Vasa. 2017; 46, 60-3.

[13] Aloufi AD, Memish ZA, Assiri A, McNabb SJ. Trends of reported human cases of brucellosis, Kingdom of Saudi Arabia, 2004-2012. J Epidemiol Glob Health. 2016; 6 (1), 11-18.

[14] Cascio A, De Caridi G, Lentini S, et al. Involvement of the aorta in brucellosis: the forgotten, life-threatening complication. A systematic review. Vector Borne Zoonotic Dis. 2012; 12 (10), 827-40.

[15] Herrick JA, Lederman RJ, Sullivan B, Powers JH, Palmore TN. Brucella arteritis: clinical manifestations, treatment, and prognosis. Lancet Infect Dis. 2014; 14 (6), 520-6. [16] Aziz S, Al-Anazi AR, Al-Aska AI A review of gastrointestinal manifestations of brucellosis. Saudi J Gastroenterol. 2005; 11 (1), 20-7.

[17] Aounallah A, Arouss A, Ghariani N, et al. Cutaneous leukocytoclastic vasculitis: about 85 cases. Pan Afr Med J. 2017; 26, 138. 